$1 \mathrm{~N} \cdot 02$

NASA Technical Memorandum 107726

$100=128$

SPATIAL ADAPTATION PROCEDURES ON TETRAHEDRAL

MESHES FOR UNSTEADY AERODYNAMIC FLOW

CALCULATIONS

RUSS D. RAUSCH

JOHN T. BATINA

HENRY T. Y. YANG

FEBRUARY 1993

N/Sก

National Aeronautics and

Space Administration

Langley Research Center

Hampton, Virginia 23681-0001

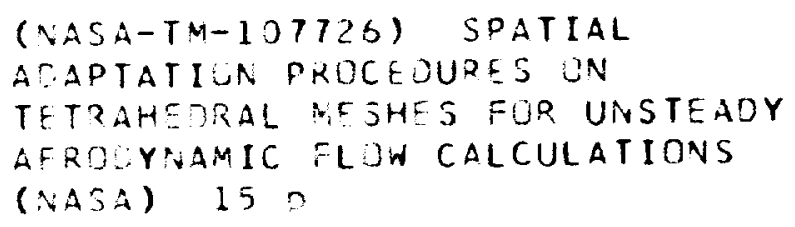

N93-22552

Unclas

$63 / 02 \quad 0150328$ 



\title{
SPATIAL ADAPTATION PROCEDURES ON TETRAHEDRAL MESHES FOR UNSTEADY AERODYNAMIC FLOW CALCULATIONS
}

\author{
Russ D. Rausch \\ Purdue University \\ West Lafayette, Indiana 47907 \\ John T. Batina ${ }^{\dagger}$ \\ NASA Langley Research Center \\ Hampton, Virginia 23681-0001
}

\author{
Henry T. Y. Yang ${ }^{*}$ \\ Purdue University \\ West Lafayette, Indiana 47907
}

\begin{abstract}
Spatial adaptation procedures for the accurate and efficient solution of steady and unsteady inviscid flow problems are described. The adaptation procedures were developed and implemented within a three-dimensional, unstructuredgrid, upwind-type Euler code. These procedures involve mesh enrichment and mesh coarsening to either add points in high gradient regions of the flow or remove points where they are not needed, respectively, to produce solutions of high spatial accuracy at minimal computational cost. The paper gives a detailed description of the enrichment and coarsening procedures and presents comparisons with experimental data for an ONERA M6 wing and an exact solution for a shock-tube problem to provide an assessment of the accuracy and efficiency of the capability. Steady and unsteady results, obtained using spatial adaptation procedures, are shown to be of high spatial accuracy, primarily in that discontinuities such as shock waves are captured very sharply.
\end{abstract}

\section{Introduction}

Considerable progress in developing methods of dynamically adapting computational meshes based on the numerical solution of partial differential equations has been made over the past decade. 'These methods are being de-

\footnotetext{
"Graduate Research Assistant, School of Aeronautics and Astronautics. Member AlAA.

'Senior Research Scientist, Unsteady Aerodynamics Branch, Structural Dynamics Division. Associate Fellow ALAA. ${ }^{\text {t}}$ Professor and Dean, Schools of Engineering. Fellow ALAA.
}

veloped to produce higher spatial accuracy in such solutions more efficiently. Spatial accuracy is obviously important when modeling continuous equations with a discrete set of points. It is generally understood that accuracy is improved when the number of mesh points in a fixed computational domain is increased. Associated with an increase in the number of mesh points, however, are increased computer run times and memory costs. Hence, for efficiency, it is important to enrich meshes locally based on the numerical solution, in contrast to using globally fine meshes, to minimize the total number of mesh points and hence minimize the cost for a given spatial accuracy. The methods of mesh refinement can be separated into three general categories: (1) mesh regeneration, (2) mesh movement, and (3) mesh enrichment.

The first method, mesh regeneration, places the work of adapting the mesh on the mesh generation program rather than on the actual numerical solution procedure of the governing equations. In this method, a solution is first obtained, and regions of relatively large discretization errors are detected. A new mesh is then generated to concentrate points in regions where the large discretization errors occur. This new mesh may contain more or fewer points than the original mesh.

For the second method, mesh movement, the number of points in the computational domain remains fixed. To improve the spatial accuracy of the solution, these points are moved into regions where solution gradients are relatively large. In general, this can be accomplished in two ways. The first way models the mesh as a spring network, where points are joined by linear springs with spring stiffnesses proportional to solution gradients. The mesh is then allowed to move into the relatively high gradient regions to produce effectively a locally finer mesh. The second way uses forcing functions in a Poisson-equation grid generator to redistribute points. Either method of mesh movement is easily imple- 
mented within existing solution algorithms because only the locations of the existing mesh points are changed.

The final method of spatial adaptation is mesh enrichment. In this method points are added to regions of relatively large solution error by dividing locally the cells which make up the mesh or by embedding finer meshes in these regions. This method differs from mesh regeneration and movement in that the mesh is made finer in local regions while the global mesh topology remains the same. The method of mesh enrichment also is generally regarded as having advantages over regeneration and movement, especially for transient problems. ${ }^{2}$ For example, a distinguishing feature of mesh enrichment is that the original mesh is recovered once a refined feature has passed. Another feature is that the procedures are relatively fast compared to remeshing the entire domain. This feature is important since spatial adaptation is performed many times to track transient flow features.

For the Euler and Navier-Stokes equations, computational fluid dynamics algorithms are being developed based on spatial adaptation methods. With these equations, relatively large spatial discretization errors may be encountered with flow features such as shock waves, shear layers, boundary layers, and expansion fans. These flow features can be resolved more accurately using the adaptation methods mentioned above. Nakahashi and Deiwert, ${ }^{3}$ for example, have used tension and torsional springs to move the mesh into regions where relatively large spatial discretization errors occur. This mesh movement approach showed considerable versatility for the problems treated. However, various constants were needed to control orthogonality and smoothness, and direct control of an optimal mesh adaptation procedure generally was not possible. Further examples of spatial adaptation methods include the work of Usab and Murman. ${ }^{4}$ In Ref. 4, embedded meshes of quadrilateral cells and nodes were used in regions of the mesh where shock waves occurred. This approach improved the spatial accuracy of the numerical method which resulted in highly accurate solutions for steady flow problems. Dannenhoffer and Baron ${ }^{5.6}$ extended the work in this area using irregularly shaped embedded regions, which were coupled to the base mesh by a multiple-grid solution algorithm. Several other examples of spatial adaptation include methods which use flow solvers based on unstructured triangular and tetrahedral meshes in two and three dimensions, respectively. Peraire et al. ${ }^{7,8}$ used mesh regeneration coupled with a finite element solution algorithm to sharply capture shock waves and complex shock structures. Lohner ${ }^{9}$ developed a procedure in three-dimensions to locally enrich the mesh for transient flow problems by dividing tetrahedral elements which make up a base mesh to capture shock waves. Further, in this procedure, elements may be removed (coarsened) from the mesh if they are not necessary to produce a given level of spatial accuracy. More recently Kallinderis, Parthasarathy, and $\mathrm{Wu}^{10}$ have developed and applied similar procedures.
With respect to solution algorithms based on unstructured meshes, the results published by the present authors demonstrated that these algorithms produce steady and unsteady solutions of comparable accuracy to results obtained using structured-grid solution algorithms. ${ }^{11}$ In Ref. 11 structured and unstructured mesh results were presented and compared for steady and unsteady flows where no mesh adaptation was used. However, in Ref. 12, the present authors demonstrated that solutions of higher spatial accuracy are indeed possible for two-dimensional steady and unsteady flows through the use of mesh adaptation. Therefore, the purpose of this paper is to report on further modifications to the three-dimensional, unstructured-grid, upwind-type Euler code reported in Refs. 13, 14, and 15 to include mesh enrichment and coarsening procedures for steady and unsteady flow calculations.

The objectives of the research are as follows: (1) to develop time-accurate mesh enrichment and coarsening procedures for spatial adaptation, (2) to test the procedures by performing steady and unsteady calculations for a variety of cases, (3) to determine the accuracy of the spatially adapted solutions by making comparisons with published solutions produced by alternative methods and existing experimental data, and (4) to assess the efficiency of the spatially adapted solutions by making comparisons of required computer resources.

The paper gives a detailed description of the mesh enrichment and coarsening procedures and gives a brief description of the solution algorithm ${ }^{13-15}$ for completeness. Steady and unsteady flow results are presented to demonstrate an application of the adaptive mesh procedures. Steady flow results are presented for the ONERA M6 wing to assess the accuracy of the computed surface pressures by making comparisons with experimental data. Unsteady flow results are presented in a three-dimensional simulation of a one-dimensional shock-tube problem to demonstrate an application of the enrichment and coarsening procedures for transient flows and to assess the accuracy of the computed results by making comparisons with the exact solution.

\section{Upwind-Type Euler Solution Algorithm}

The Euler equations are solved using the threedimensional, unstructured-grid, upwind-type solution algorithm developed by Batina. ${ }^{13}$ The solution algorithm of Ref. 13 was extended by the present authors ${ }^{14,15}$ for time-accurate, unsteady flow calculations on a deforming mesh. The algorithm, which is a cell-centered, finitevolume scheme, uses upwind differencing based on fluxvector splitting, ${ }^{16}$ similar to upwind schemes developed for use on structured meshes. The flux-split discretization accounts for the local wave-propagation characteristics of the flow and captures shock waves sharply with at most one grid point within the shock structure. An additional advantage of 
using flux-splitting is that the discretization is naturally dissipative and, consequently, does not require additional artificial dissipation terms or the adjustment of free parameters to control the dissipation. However, in calculations involving a higher-order upwind scheme such as this, oscillations in the solution near shock waves are expected to occur. To eliminate these oscillations, flux limiting usually is required. In the present study, a continuously differentiable flux limiter was employed.

The Euler equations are integrated in time using an implicit time-integration scheme involving a Gauss-Seidel relaxation procedure. ${ }^{13}$ The relaxation procedure is implemented by re-ordering the elements that make up the unstructured mesh from upstream to downstream. The solution is obtained by sweeping two times through the mesh as dictated by stability considerations. The first sweep is performed in the direction from upstream to downstream, and the second sweep is from downstream to upstream. For purely supersonic flows, the second sweep is unnecessary. This relaxation scheme is stable for large time steps and thus allows the selection of the step size based on the temporal accuracy of the problem being considered, rather than on the numerical stability of the algorithm. Consequently, very large time steps may be used for rapid convergence to steady state, and an appropriate step size may bc selected for unsteady cases, independent of numerical stability issues.

\section{Spatial Adaptation Procedures}

In this section, the spatial adaptation procedures are described. These descriptions include detailed explanations of the procedures used to detect flow features and the procedures used to enrich and coarsen meshes of tetrahedra.

\section{Flow Feature Detection}

The first step of the spatial adaptation procedures is the detection of regions of relatively large discretization error so that the computational mesh can be locally enriched to improve the spatial accuracy or coarsened locally to reduce the computational costs. For numerical solutions of the Euler equations, these regions generally occur near flow features such as shock waves, stagnation points, slip lines, and expansion fans. The dominant flow feature for the cases considered in this study is a shock wave.

There are a number of flow parameters that can be used for enrichment indicators based on the detection of shock waves. Parameters such as density, pressure, or total velocity are useful since these quantities are discontinuous through shocks. For example, first or second, divided or undivided differences in one of these parameters, similar to the work by Dannenhoffer and Baron, ${ }^{6}$ can be used to detect shock waves. The enrichment indicator used in this study was the magnitude of the density gradient $|\nabla \rho|$ which is often used to detect shock waves for steady flow problems.

\section{Mesh Enrichment}

Mesh enrichment of tetrahedral meshes is performed by starting with a relatively coarse mesh of cells and then subdividing these cells until a given level of spatial accuracy has been obtained. To prevent cells from being enriched too many times near flow discontinuities such as shock waves, an upper bound is placed on the number of times a cell can be divided. Presently, the upper bound is set before a calculation is performed where the upper bound is usually constrained by the computer memory available. For transient problems the mesh enrichment procedure may be performed at each time step of the integration of the governing flow equations or it may be performed once every set number of time steps.

There are a number of ways to subdivide a tetrahedral cell. For example, a node could be added at the centroid of a cell and subdivided accordingly. This way, however, is not appealing because it often produces irregular cells, which tend to have an adverse effect on the accuracy of the solution algorithm. Another way is to add nodes at arbitrary locations along the edges of a cell. This is the approach taken in this study, but for convenience and to maintain regular cells, the nodes are added at the midpoints of the edges of the cells.

Mesh enrichment is performed by using the enrichment indicator to determine if a cell is to be subdivided into smaller cells. To accomplish this, the enrichment indicator is computed for each cell and compared with a threshold value to determine whether a cell should be subdivided. In this study, the threshold value is set before the calculation is performed. If the threshold is exceeded, a new node is created at the midpoint of each edge of the tetrahedral cell, and the cell is subdivided into eight smaller cells. Special care must be taken, however, when an edge that is to be bisected lies on a boundary of the mesh, since the midpoint of the edge does not generally lie on the boundary. In this case, the location of the new node is determined by using a spline of the boundary coordinates. Further, the values of the flow variables for the new cells are determined by a linear interpolation of the conserved variables located at the nodes of the original cells.

For a given tetrahedral cell to be enriched, either one edge, three edges (all part of the same triangular face), or all six edges are bisected. In the event that only two edges are marked to be bisected and they are on the same face of the cell, the third edge of the face is automatically bisected to prevent the creation of highly skewed or stretched cells. Similarly, if four or five edges of the cell are marked to be bisected, the remaining edges are bisected and the cell is fully enriched. Each time the mesh is enriched, a cell may be divided in one of three ways. The first way, shown 


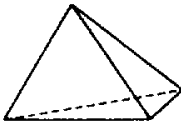

(a)

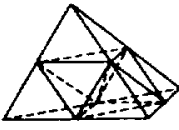

(b)

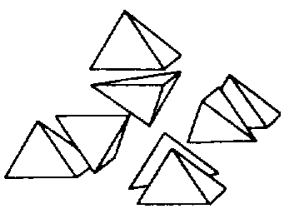

(c)
Fig. 1 Diagram of a type-8 element enrichment.

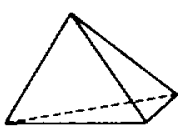

(a)

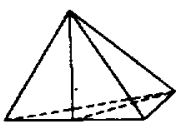

(b)

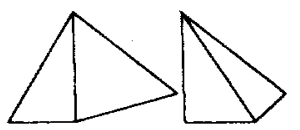

(c)
Fig. 2 Diagram of a type-2 element enrichment.

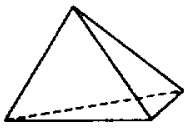

(a)

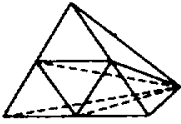

(b)
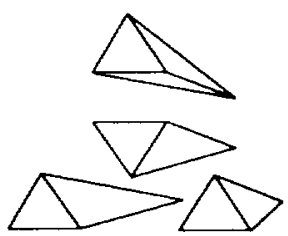

(c)
Fig. 3 Diagram of a type-4 element enrichment.

in Fig. 1, results when all six edges of a cell have been marked to be bisected. In this situation the cell is divided into eight new cells where the vertices of the inner cells are, in general, midpoints of edges that make up the original cell. The original cell is thus referred to as a type- 8 element since after enrichment it becomes eight new tetrahedral cells. It should be noted that this way of subdividing a tetrahedron is not unique because there are three possible choices for the orientation of the inner diagonal edge that passes through the original cell. In the procedures, the orientation of the inner diagonal is based solely on the numbering of the nodes for the original cell being enriched. The second way, shown in Fig. 2, occurs if only one edge of the original cell is marked to be bisected. In this situation, the marked edge is bisected, and two new cells are formed. The original cell is thus referred to as a type- 2 transition element since after enrichment it becomes two new cells. The third and final way, shown in Fig. 3, occurs if all three edges of a single face of the base cell are marked to be bisected. In this situation, the marked edges are bisected, and four new cells are formed. The original cell is thus referred to as a type- 4 transition element since after enrichment it becomes four new cells.

New cells formed from a type- 8 element may be enriched further. However, to prevent highly stretched cells, type- 2 or type- 4 transition elements are restricted from being divided further as indicated in Figs. 4 and 5. For cells from a type- 2 transition element, if any of the nine edges that make up the two new cells are marked for enrichment, the original cell is made into a type-8 element as shown in Fig. 4. If in addition to this, either or both of the bottom two edges are marked (the lower left, right, or both), cells of the type-8 el-
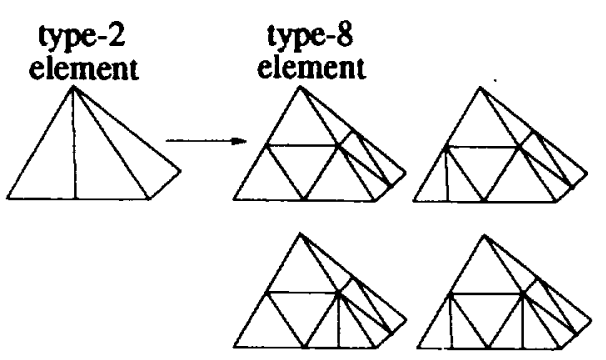

Fig. 4 Diagram illustrating details of further enrichment of type-2 transition elements.

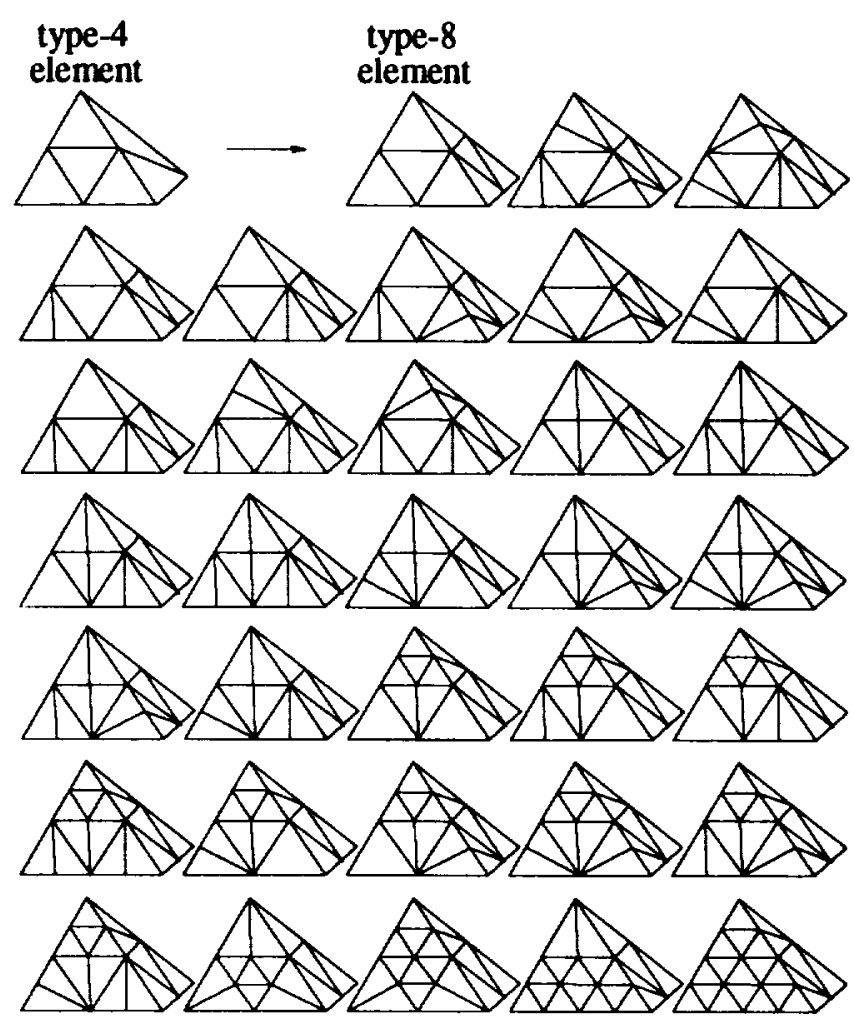

Fig. 5 Diagram illustrating details of further enrichment of type -4 transition elements.

ement are further enriched accordingly, as shown in Fig. 4. Similarly for cells from a type-4 transition element, if any of the fifteen edges that make up the four cells are marked to be bisected, the original cell is also made into a type-8 element as shown in Fig. 5. If further, any edge that is part of the type- 4 transition face is marked to be bisected, cells of the type-8 element are additionally enriched, as shown in Fig. 5. The type-4 transition face has nine edges that may be bisected further. If there is not a restriction on the edges marked to be bisected, a total of 512 permutations of further enrichment could result. However, since a triangular face of a type-4 transition element is prevented from having only two of its three edges marked for bisection, the total number of possible permutations is reduced to 89 . Eliminating permutations that are similar when rotated results in the 33 enriched cells shown in Fig. 5. 


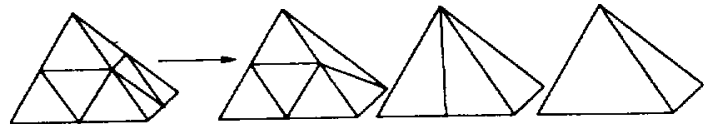

(a) type-8 element coarsening.

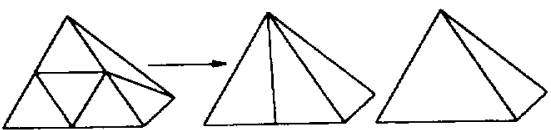

(b) type-4 transition element coarsening.

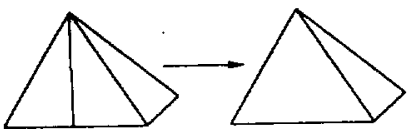

(c) type-2 transition element coarsening.

Fig. 6 Diagrams illustrating mesh coarsening possibilities.

\section{Mesh Coarsening}

Mesh coarsening of tetrahedral meshes is performed by removing added nodes and cells from previously enriched meshes to delete them from local regions of the mesh where certain flow features are no longer present. This procedure is necessary to adapt meshes to the numerical solution of the governing flow equations in order to minimize computational cost. Candidate cells for removal are cells that came from type-2, type-4, or type-8 elements and were marked for removal. Each time the mesh is coarsened, cells and nodes may be removed in one of several ways as shown in Fig. 6 . For a type- 8 element, three, five, or six nodes may be removed resulting in a type-4, a type-2, or an original cell, respectively (Fig. 6(a)). Similarly, if two of the three nodes that form the inner triangle of the face of a type-4 element are candidate nodes, the two nodes are removed and a type2 element is formed as shown in Fig. 6(b). Likewise, if all three nodes that form the inner triangle of the face of a type4 element are candidate nodes, the three nodes are removed and the one original cell that was divided previously into four remains (Fig. $6(\mathrm{~b})$ ). However, if only one of the three nodes that form the inner triangle of the subdivided face of a type-4 element is a candidate node then nothing is done. For a type-2 element there is only one node that may be removed which is the midpoint of a previously bisected edge. Removal of this node leaves only the cell that was divided originally into two as shown in Fig. 6(c). It should be noted that the mesh cannot become coarser than the original mesh.

\section{Results and Discussion}

Adaptive mesh results are presented in this section for a test case involving a simulated flow field using a mesh generated inside a cube, an ONERA M6 wing, and a three-dimensional simulation of a one-dimensional shocktube problem. The results are used to assess the spatial adaptation procedures in three dimensions. The accuracy of the results are determined by making comparisons with

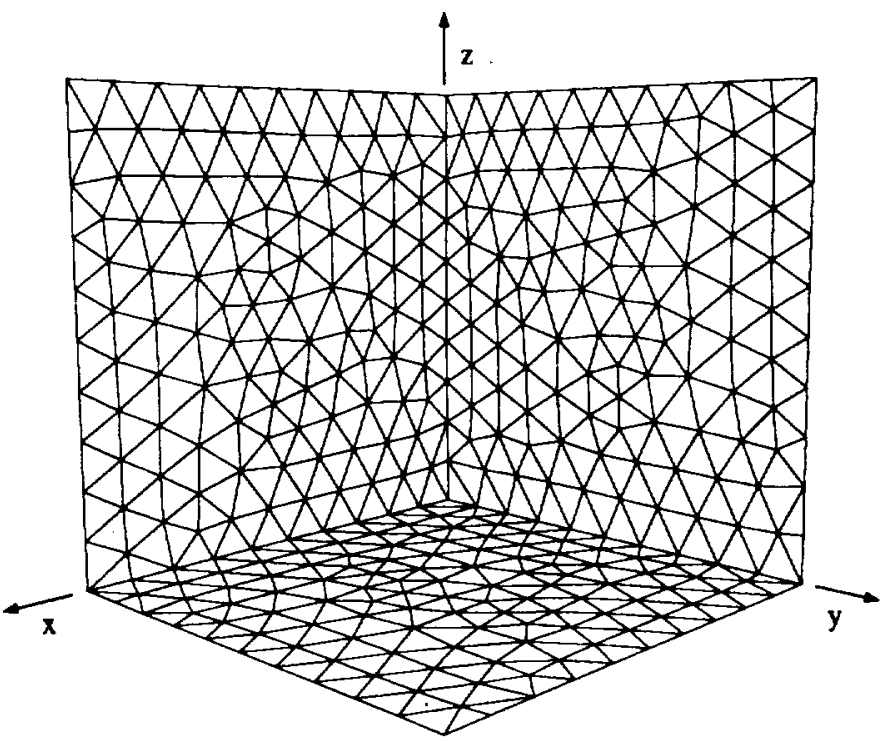

Fig. 7 Partial view of the surface of the original coarse mesh used in test case of the spatial adaptation procedures (1,805 nodes, 8,557 tetrahedra).

results from alternative methods and available experimental data.

\section{Test Case for the 3D Adaptive Mesh Procedures}

To demonstrate the spatial adaptation procedures in three-dimensions a test case was performed. The test case was devised to assess newly developed procedures and data structures implemented within the mesh enrichment and coarsening procedures. The test case involves a mesh generated inside a unit cube, where a partial view of the surface mesh is shown in Fig. 7. The mesh was generated using the advancing front mesh generation package, VGRID3D. ${ }^{17}$ In Fig. 7 three sides of the surface mesh have been removed so that the interior of the mesh can be seen. The total mesh contains 1,805 nodes and 8,557 tetrahedra and serves as a starting mesh for the adaptive mesh procedures.

The test case simulates an unsteady problem to demonstrate the mesh coarsening procedures. This case involves subdividing cells in the vicinity of the surface of a sphere, were the radius $r$ of the sphere is increased with time at a constant rate. The equation for the surface of the sphere is given by

$$
x^{2}+y^{2}+z^{2}=r^{2}
$$

where the origin of the sphere is the corner of the mesh. This equation is used to specify values of a field variable, $\psi$, for each cell in the mesh. For example, the value of $\psi$ for a cell is zero if the centroid of the cell lies within $\left(x^{2}+y^{2}+z^{2}<r^{2}\right)$ the sphere and the value of $\psi$ for a cell is one if the centroid of the cell lies outside $\left(x^{2}+y^{2}+z^{2}>r^{2}\right)$ the sphere. These values of $\psi$ are used to compute the magnitude of the gradient of $\psi$ for 


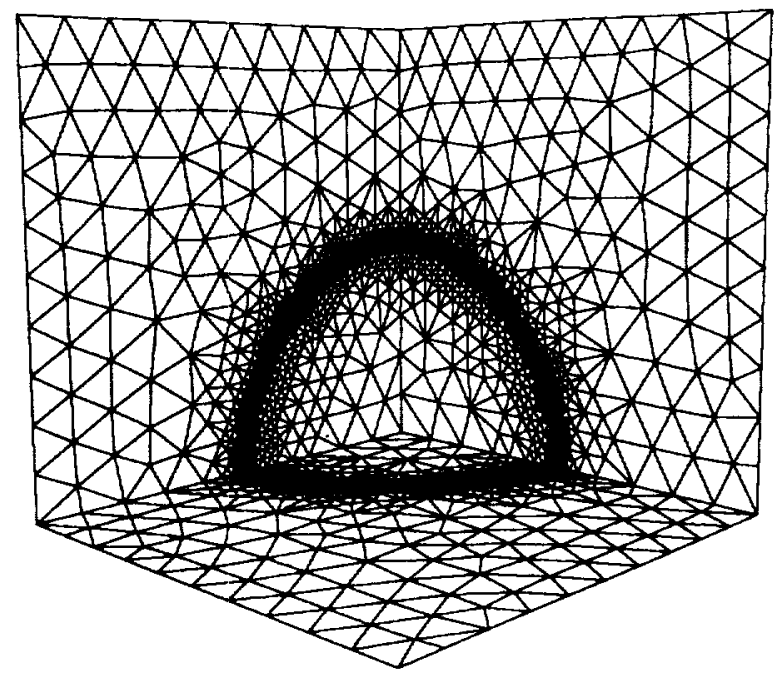

$r=0.50(23,120$ nodes, 129,795 tetrahedra $)$.

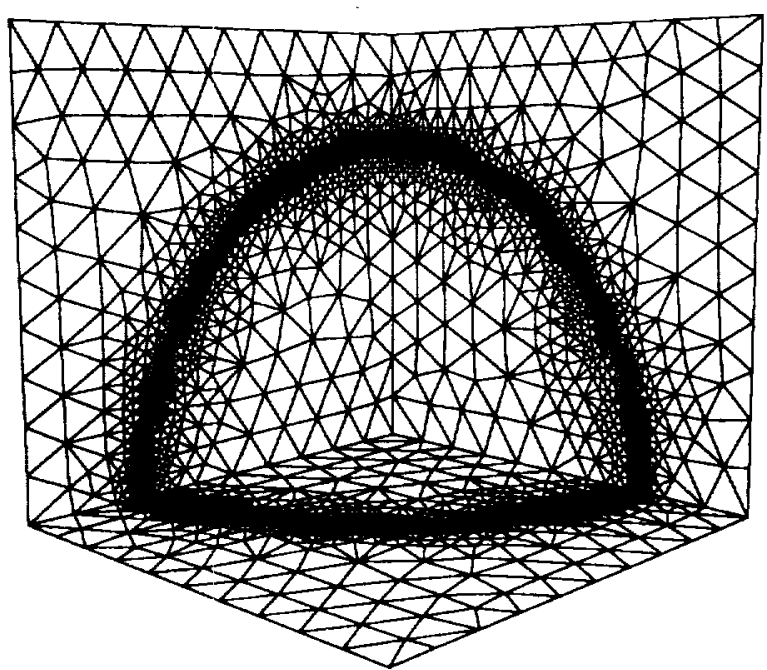

$r=0.75(49,335$ nodes, 281,820 tetrahedra $)$.

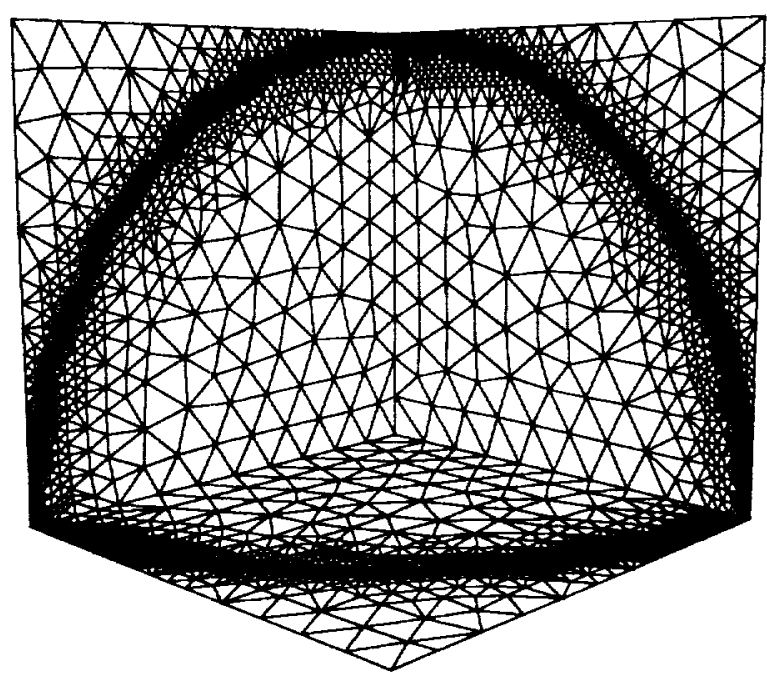

$r=1.00(85,462$ nodes, 489,369 tetrahedra $)$.

Fig. 8 Sequence of instantaneous surface meshes for the unsteady test case using three levels of enrichment.

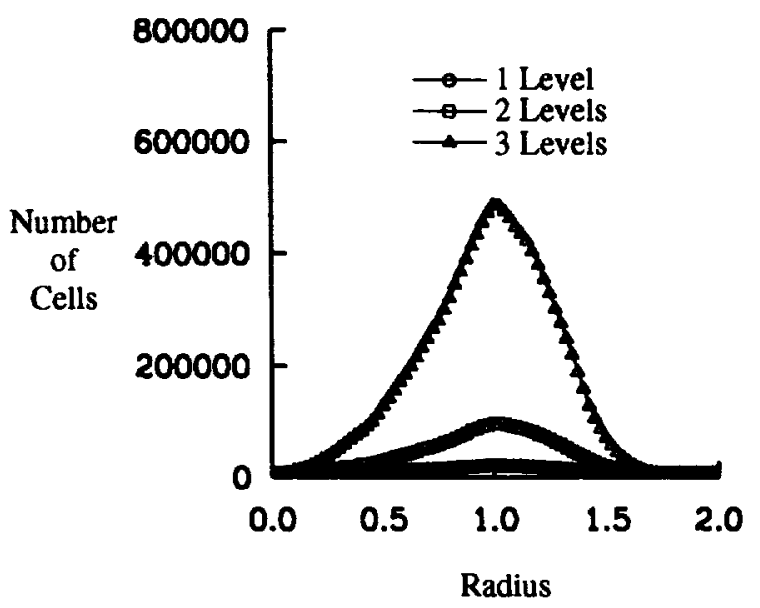

Fig. 9 Variation of the number of tetrahedral cells in the mesh for the unsteady test case using 1 , 2 , and 3 levels of enrichment.

each cell in the mesh, where the magnitude of $\nabla \psi$ is used as the enrichment indicator. For the test case, three calculations were performed allowing one, two, and three levels of enrichment. For each calculation the spherical wave propagates in increments of $\Delta r=0.025$ of the radius for every time step and the calculation was continued until the radius $r$ was 2.0. The sequence of surface meshes for the third calculation with three levels of enrichment is shown in Fig. 8 at three moments in time corresponding to a radius $r$ of $0.50,0.75$, and 1.00 . In this figure the surface meshes sharply define the spherical wave yet the adapted regions transition smoothly to the coarser regions of the mesh. A plot of the variation of the number of tetrahedral cells in the mesh versus the radius of the spherical wave is shown in Fig. 9 for the three calculations performed. In each calculation the number of cells in the mesh varies smoothly as the spherical wave propagates through the mesh. This figure illustrates the rapid increase in the number of cells in the mesh as the enrichment levels are increased, which may be surprising for such a simple test case.

\section{ONERA M6 Wing}

The ONERA M6 wing was selected to further assess the adaptive mesh procedures in three dimensions. The accuracy of the adapted mesh results are assessed by making comparisons with experimental data and by making comparisons with results obtained from other unstructured-grid Euler codes. The M6 wing has an aspect ratio of 3.8, a leading edge sweep angle of $30^{\circ}$, and a taper ratio of 0.56 . The airfoil section is the ONERA D airfoil, which is a symmetric $10 \%$ maximum thickness-to-chord ratio conventional section. The wing tip is rounded and is defined by a half body of revolution of the airfoil section. This wing has been widely studied and results have been obtained using many flow solvers on both structured ${ }^{18}$ and unstructured ${ }^{19-21}$ meshes. A steady flow calculation was performed at a 


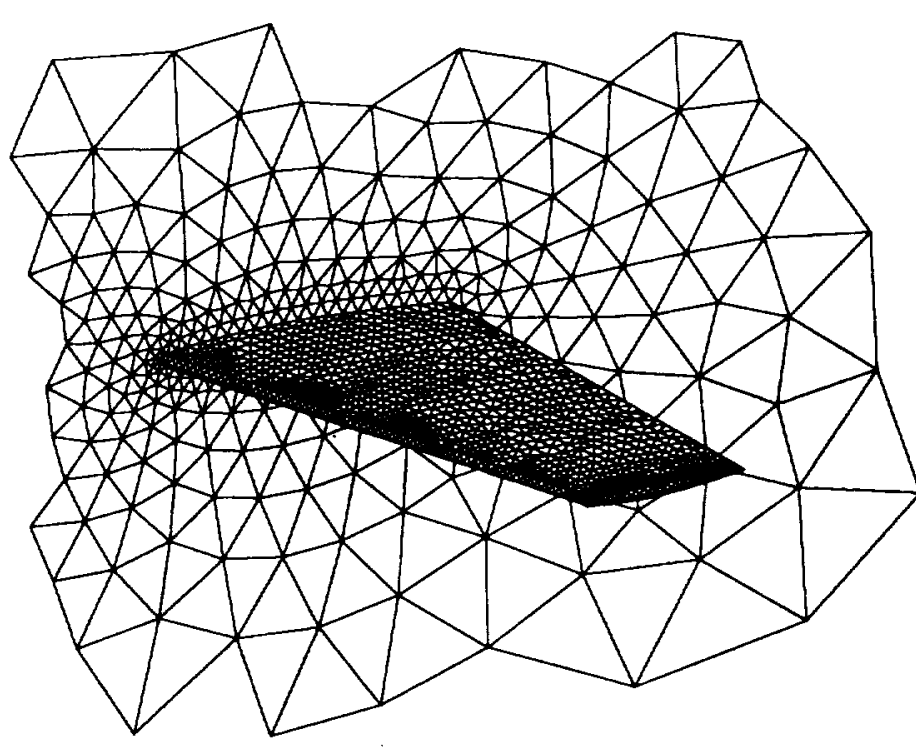

Fig. 10 Partial view of the original coarse surface mesh of the symmetry plane and the ONERA M6 wing.

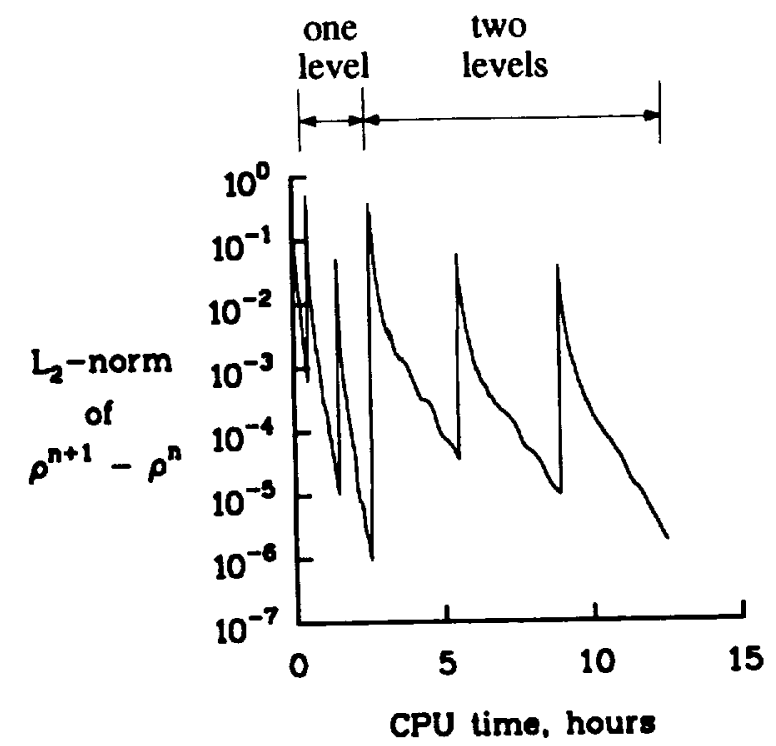

Fig. 11 Steady-state convergence history for the ONERA M6 wing at $M_{\infty}=0.84$ and $\alpha_{0}=3.06^{\circ}$ using the adaptive mesh procedures.

freestream Mach number of 0.84 and an angle of attack of $3.06^{\circ}$. For this case, the starting mesh and the spatially adapted meshes are presented along with the corresponding density contour lines. Additionally, surface pressures were obtained for comparison with experimental data reported by Schmidt and Charpin. ${ }^{22}$

The mesh about the ONERA M6 wing configuration was generated using VGRID3D. The mesh extends $2 \frac{1}{2}$ wing semispans from the symmetry plane in the span direction. Also, the mesh extends $6 \frac{1}{2}$ root chordlengths above/below the wing surface as well as $6 \frac{1}{2}$ root chordlengths upstream and 10 root chordlengths downstream of the wing to rectan-

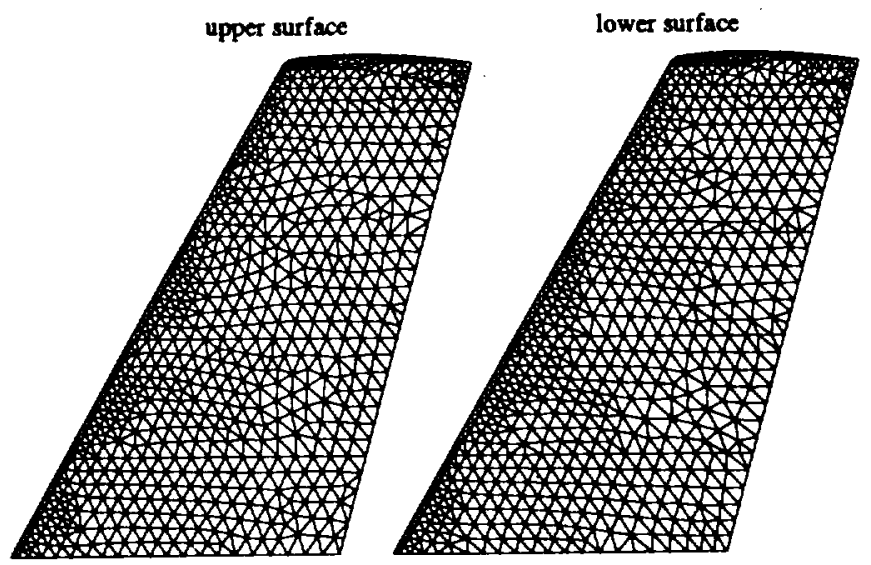

(a) Original mesh.

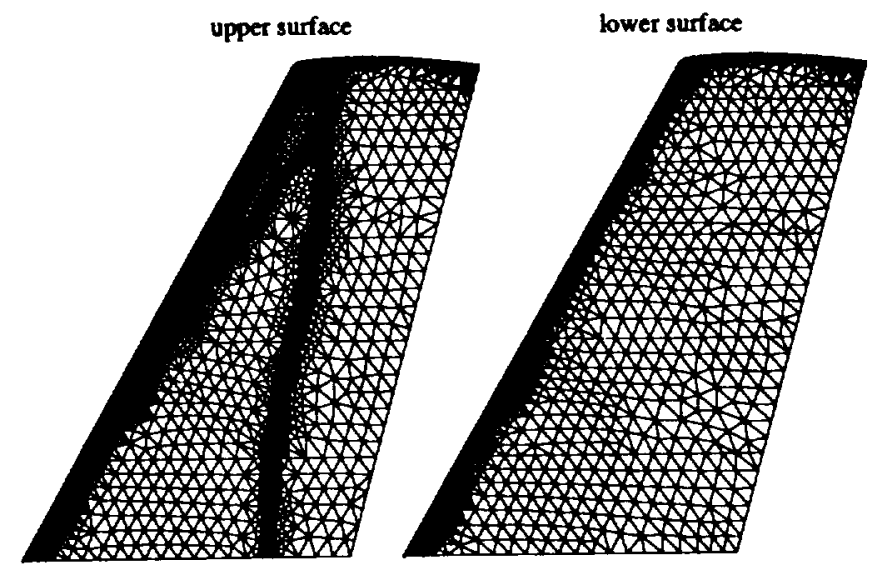

(b) Two enrichment levels.

Fig. 12 Upper and lower surface meshes of the ONERA M6 wing for the original and adapted meshes.

gular outer boundaries. The complete coarse mesh for the M6 wing contains 8,824 nodes and 46,516 tetrahedral cells. A partial view of the surface mesh for the symmetry plane and wing is shown in Fig. 10.

The calculation was performed using implicit time integration at a $C F L$ number of 100,000 . The final solution was obtained by adapting the mesh to the magnitude of the density gradient every 300 iterations for the first 1,500 iterations and then marching the solution an additional 300 iterations on the final adapted mesh. Also, the adaptive mesh procedures allowed only one level of enrichment during the first 900 iterations, and two levels were allowed thereafter. The convergence history for the calculation is shown in Fig. 11 where the $L_{2}$-norm of the density residual is plotted versus the CPU time in hours. In Fig. 11 the spikes in the $L_{2}$-norm of the density residual mark the iterations at which spatial adaptation was performed.

Figure 12 shows the upper and lower surface meshes for the original starting mesh and the final adapted mesh using two levels of enrichment. A summary of the sizes of the original and adapted meshes are given in Table 1. The information in the table consists of the total number of 


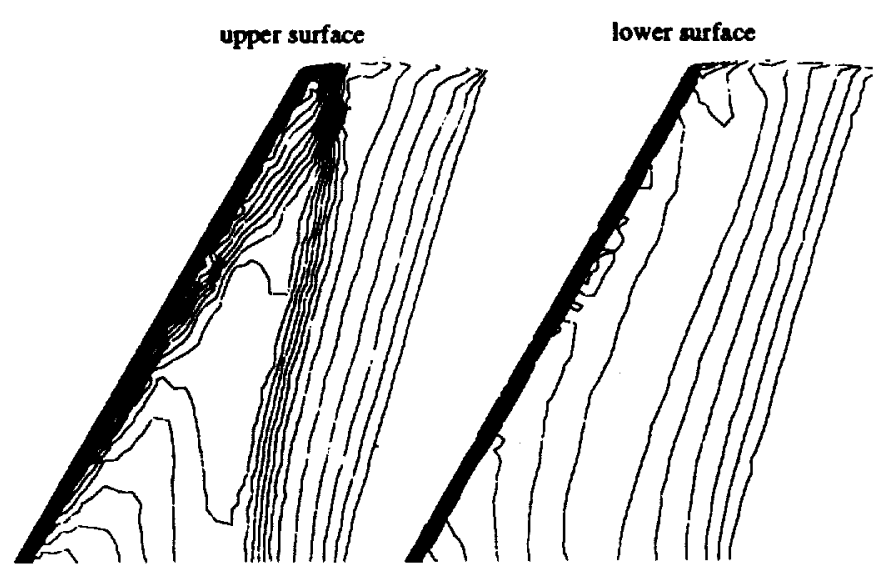

(a) Original mesh.

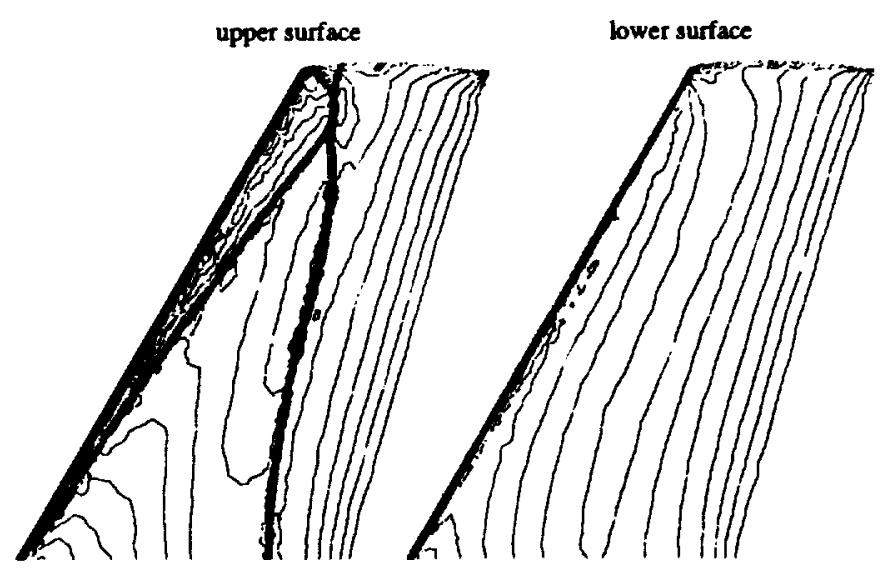

(b) Two enrichment levels.

Fig. 13 Upper and lower surface density contour lines $(\Delta \rho=0.025)$ for the ONERA M6 wing at $M_{\infty}=0.84$ and $\alpha_{0}=3.06^{\circ}$ using the original and adapted meshes.

cells, nodes, boundary faces, wing boundary faces, and wing boundary nodes for each mesh. Notice in Table 1 that after one level of enrichment, the number of cells in the mesh is approximately twice as large as the number of cells in the original mesh. After two levels of enrichment, the mesh is

Table 1 Comparisons of the ONERA M6 wing meshes.

\begin{tabular}{lccc}
\hline & $\begin{array}{c}\text { Original } \\
\text { Mesh }\end{array}$ & $\begin{array}{c}\text { One } \\
\text { Level }\end{array}$ & $\begin{array}{c}\text { Two } \\
\text { Levels }\end{array}$ \\
\hline Number of Cells & 46,516 & 105,876 & 352,417 \\
Number of Nodes & 8,824 & 19,728 & 63,855 \\
$\begin{array}{l}\text { Number of Boundary } \\
\text { Faces }\end{array}$ & 4,190 & 7,606 & 17,362 \\
$\begin{array}{l}\text { Number of Wing } \\
\text { Boundary Faces }\end{array}$ & 2,792 & 6,048 & 15,578 \\
$\begin{array}{l}\text { Number of Wing } \\
\text { Boundary Nodes }\end{array}$ & 1,419 & 3,052 & 7,822 \\
\hline
\end{tabular}

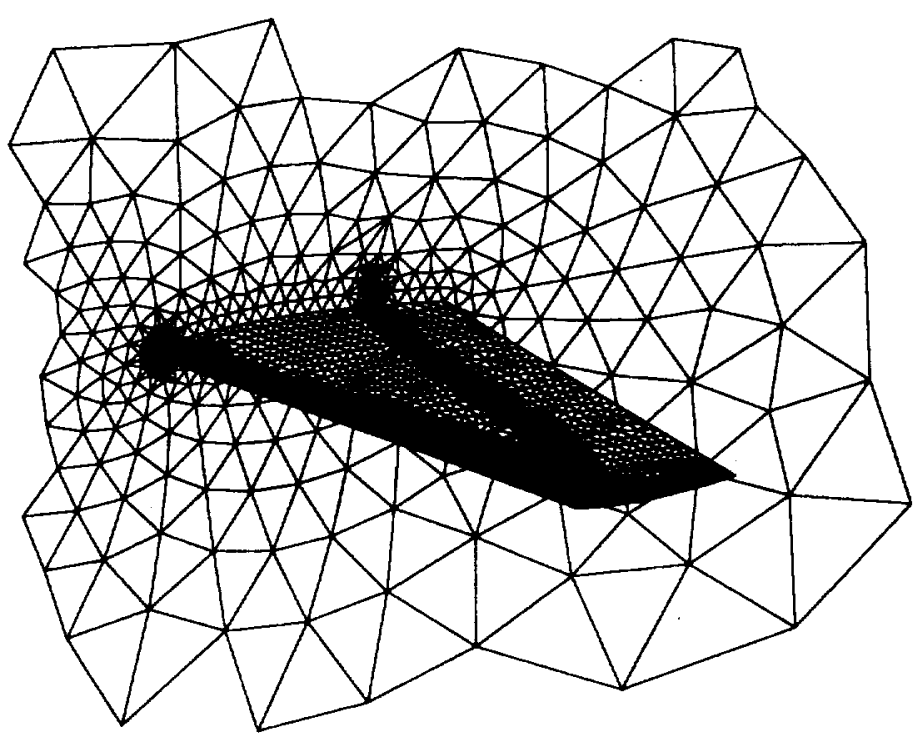

Fig. 14 Partial view of the adapted surface mesh of the symmetry plane and the ONERA M6 wing at $M_{\infty}=0.84$ and $\alpha_{0}=3.06^{\circ}$.

7.6 times larger. This increase in the number of cells is significant since it directly affects the computational resources required to perform a given calculation using the adapted meshes. Figure 13 shows the surface density contour lines on the original and adapted meshes using an increment of $\Delta \rho=0.025$. In Figs. 12 and 13 the upper surface is shown to the left and the lower surface is shown to the right. The upper surface contours (left part of Fig. 13) clearly show the lambda-shaped shock pattern formed by the two inboard shocks that coalesce near $87 \%$ semispan and then separate just outboard of $95 \%$ semispan (shown clearly for two levels of enrichment). The forward shock of the two shock region is a supersonic to supersonic shock. The aft shock of the two shock region is a supersonic to subsonic shock. The lower surface contours (right part of figure) indicate that there is little spanwise variation in density. A comparison of the two sets of contour lines reveals a considerable improvement in the resolution of the shocks when spatial adaptation is used. The meshes for the corresponding surface density contours show that points were clustered in the shock regions to produce shock waves that are spatially sharp. Also, points were clustered near the leading edge to improve the accuracy of this high gradient region. A partial view of the surface mesh and density contour lines for the symmetry plane and wing are shown in Figs. 14 and 15. In Fig. 14 the mesh in the symmetry plane gives an indication of the mesh spacing off of the surface of the wing. Similarly, in Fig 15 the contour lines give an indication of the spatial resolution of the solution off of the surface of the wing.

To assess the accuracy of the results, the calculated surface pressure coefficients are compared to the experimental data at six semispan stations for the original and adapted meshes. The semispan stations are at $\eta=0.20,0.44,0.65$, 


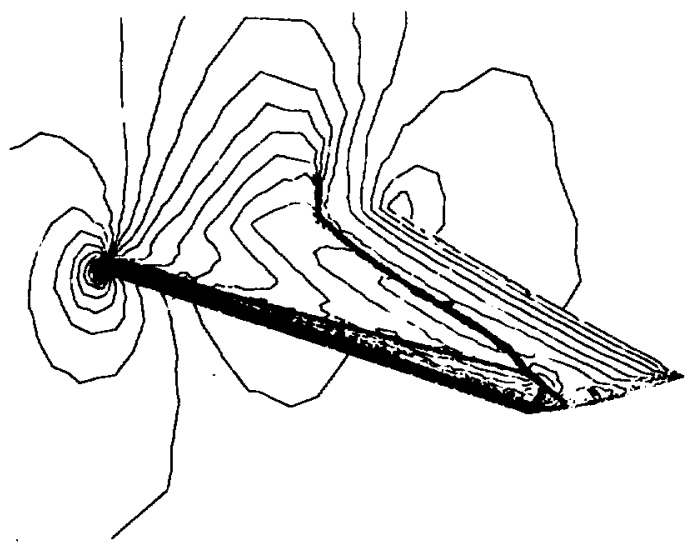

Fig. 15 Partial view of the surface density contour lines $(\Delta \rho=0.025)$ on the symmetry plane and on the ONERA M6 wing at $M_{\infty}=0.84$ and $\alpha_{0}=3.06^{\circ}$.
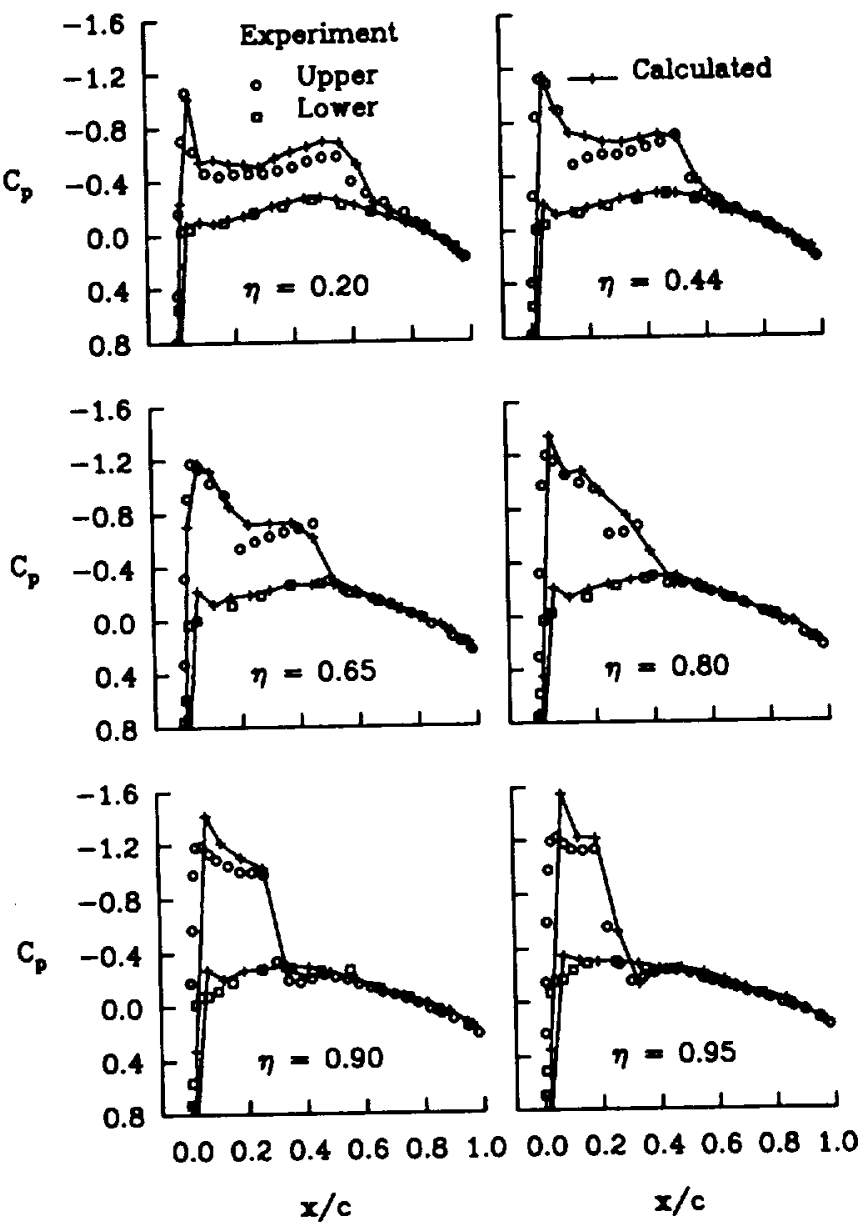

Fig. 16 Comparison of calculated and experimental values of pressure coefficient computed using the original coarse mesh for the ONERA M6 wing at $M_{\infty}=0.84$ and $\alpha_{0}=3.06^{\circ}$.
$0.80,0.90$, and 0.95 . Here the upwind-biased flow variables of surface triangles, with a common edge along the semispan station, are interpolated to the edge to determine the surface pressures. The comparisons of surface pressures are shown in Figs. 16 and 17 for the original and the adapted mesh involving two levels of enrichment. In these figures the calculated Euler results are given by solid curves where plus symbols have been used to mark the interpolated values along the semispan stations. The experimental data is denoted by circle and square symbols representing upper and lower surface pressure coefficient data. A comparison of the calculated surface pressures in Figs. 16 and 17 shows an improvement using the adapted mesh (Fig. 17), especially on the upper surface near the leading edge where the suction peak was poorly predicted in Fig. 16 using the coarser mesh. For Fig. 17 at $\eta=0.20$ there are two shock waves on the upper surface. The forward shock is well predicted and agrees well with the data. The aft shock wave is too strong and located too far back on the wing when compared with the data. This however is consistent with other inviscid flow
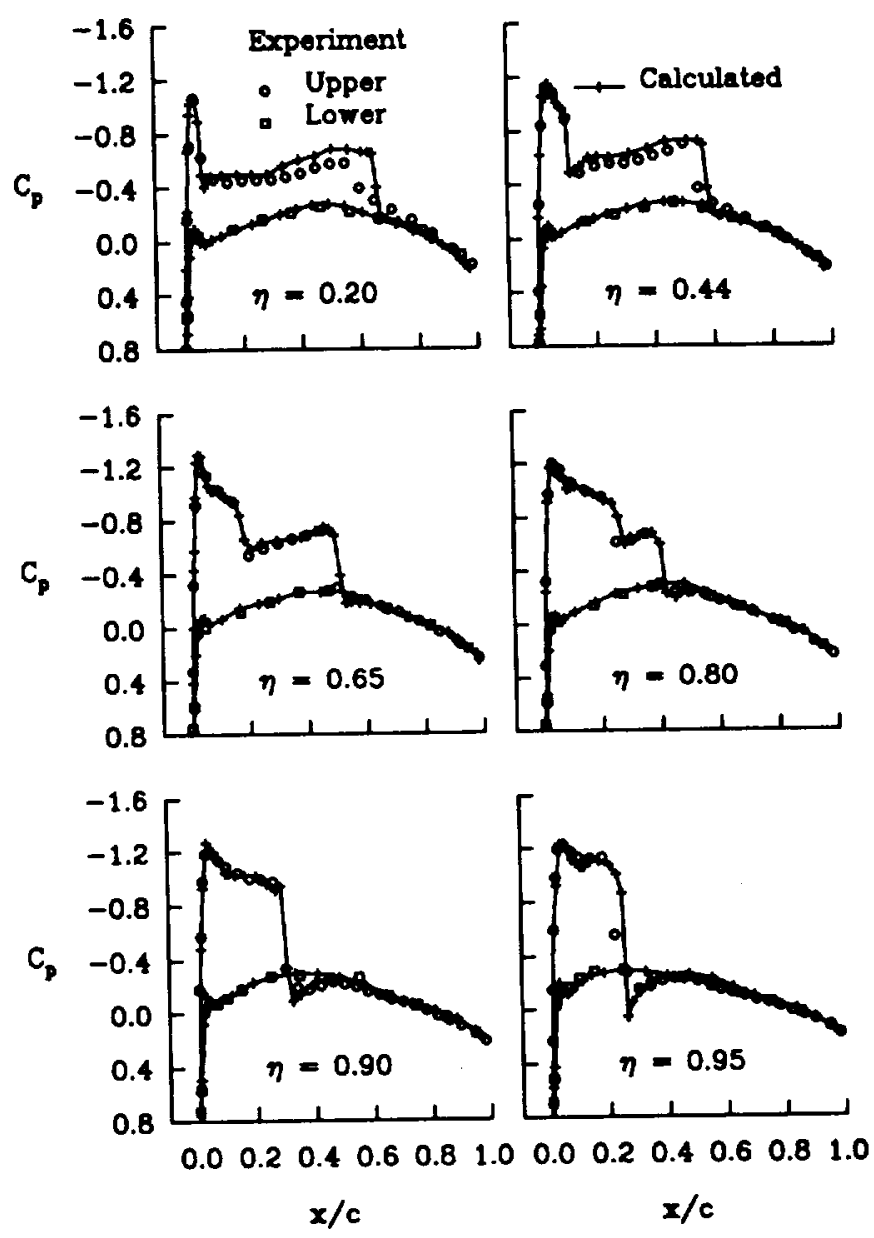

Fig. 17 Comparison of calculated and experimental values of pressure coefficient computed using the adapted mesh ( 2 levels) for the ONERA M6 wing at $M_{\infty}=0.84$ and $\alpha_{0}=3.06^{\circ}$. 
calculations for this case. At $\eta=0.44$ the forward shock has moved aft and the aft shock has moved forward. Again the forward shock wave is well predicted and the aft shock is slightly strong in comparison with the data. At $\eta=0.65$ the forward shock is near $19 \%$ chord and the aft shock wave is near $50 \%$ chord. Both shock waves are well predicted, and the pressure level between the two shock waves agrees well with the data. At $\eta=0.80$ the shock waves have begun to coalesce near $30 \%$ chord. A feature of this data worth noting is that the spatial adaptation procedures have helped to clearly define the two shock waves at $\eta=0.80$. Calculations by other researchers ${ }^{18-20}$ have failed to isolate the two shock waves at this semi-span location mainly due to the coarsness of the meshes used. At $\eta=0.90$ the two shock waves have merged to form a single strong shock near $30 \%$ chord. The shock wave is sharply captured with one interior grid point, which is common for upwind schemes of this type. Finally, at $\eta=0.95$ the upper surface pressure results show a strong shock slightly aft of the experimental data. Also, just aft of the leading edge, the upper surface results slightly underpredict the data. In general the lower surface pressure coefficients agree well with the data at all semispan stations.

The computed results are further assessed by comparing the lift coefficients obtained using the original mesh and the adapted mesh (two levels of enrichment) with those reported in Refs. 20, 23, and 24. This comparison is shown in Table 2, where the lift coefficient in Ref. 20 was computed on a mesh with 53,989 nodes and 288,170 cells, the lift coefficient in Ref. 23 was computed on a mesh with 16,984 nodes and 231,507 cells, and the lift coefficient in Ref. 24 was computed on a mesh with 173,412 nodes and $1,013,718$ cells. For these values of lift coefficient a reference area of $S_{\text {res }}=0.5255$ was used. The table shows good agreement between the lift on the final adapted mesh (two levels) and the lift obtained using the other unstructured Euler solvers giving confidence in the adapted mesh solution.

The calculation for the final adapted mesh solution was run for 1,800 iterations and required approximately 12.5 hours of CPU time and $125 \mathrm{mw}$ of memory on the Cray2 computer at the NAS facility located at the NASA Ames Research Center.

Table 2 Comparisons of lift coefficients for the ONERA M6 wing at $M_{\infty}=0.84$ and $\alpha_{0}=3.06^{\circ}$.

\begin{tabular}{lccccc}
\hline & Original & Two & Ref. & Ref. & Ref. \\
& Mesh & Levels & 20 & 23 & 24 \\
\hline Lift Coef. & 0.2827 & 0.2901 & 0.2923 & 0.2911 & 0.2901 \\
\hline
\end{tabular}

\section{Shock-Tube Problem}

An unsteady one-dimensional shock-tube problem was used to evaluate the accuracy and efficiency of the spatial adaptation procedures in three-dimensions. The shock-tube problem is illustrated in Fig. 18 where a diaphragm sepa-

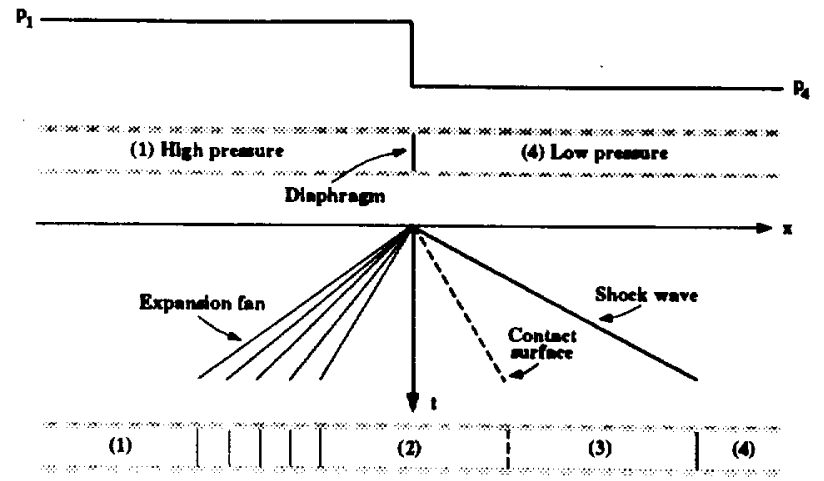

Fig. 18 Illustration of a shock-tube problem.

rates a high pressure (compression) chamber (1) and a low pressure (expansion) chamber (4). Initially the pressure distribution in the shock-tube is an ideal "step". At the instant the diaphragm bursts the initial pressure "step" separates into a shock wave, which propagates to the right into the low pressure chamber, and an expansion fan, which propagates to the left into the high pressure chamber. The region traversed by the shock (3) and the region traversed by the expansion fan (2) is separated by a contact surface. Each interface between the four regions moves at a constant speed as shown in Fig. 18.

The shock-tube problem provides a good test case for the adaptive mesh procedures since the flow contains many features expected to occur in transient problems such as a moving shock wave, an expansion fan, and a contact surface. Also, the exact solution ${ }^{25}$ is available for comparison, and the computational resources for this problem are relatively small. The problem is a challenging one for the spatial adaptation procedures since all the flow features must be tracked accurately in time as the solution progresses.

The test case considered involves air at the same temperature in the low and high pressure chambers and a diaphragm pressure ratio of five. The initial values of the primitive variables on each side of the diaphragm are given below in Eq. 2.

$$
\begin{aligned}
& \frac{\rho_{1}}{\rho_{4}}=5 \\
& u_{1}=v_{1}=w_{1}=0 \\
& u_{4}=v_{4}=w_{4}=0 \\
& \frac{p_{1}}{p_{4}}=5
\end{aligned}
$$

The calculations were performed on a coarse mesh, within parallelepiped boundaries, of unit length and with width and height that is four percent of the length of the tube. The mesh was generated using the VGRID3D mesh generator. Figure 19 shows the surface mesh for the parallelepiped. The total mesh contains 1,800 tetrahedra and 562 nodes.

The first calculation for the shock-tube problem was performed on the coarse mesh to obtain a solution for comparison with the exact solution. The coarse mesh result 


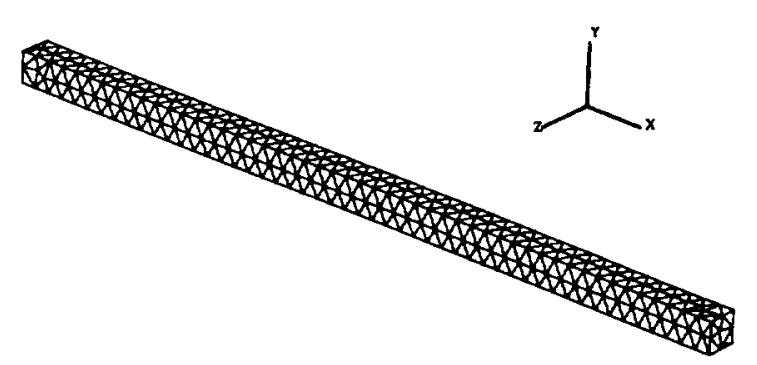

Fig. 19 Surface mesh for the parallelepiped.

also provides a solution for comparison with the spatially adapted results. The solution was obtained using implicit time-marching with a nondimensional time step of 0.001 . The calculated density profiles at three moments in time, 0.1 , 0.2 , and 0.3 are shown in Fig. 20. These profiles were obtained by sorting all 1,800 cell-centered values of the primitive variables according to their $\mathbf{x}$-coordinate locations and plotting every other point. Since the three-dimensional solution is plotted as a function of the $x$-coordinate there may be a variation of the solution in the $y$ - and z-coordinate directions. As the solution in Fig. 20 progresses in time, the expansion fan spreads and moves to the left while the shock, followed by the contact surface, moves to the right. Figure 21 shows the surface density contour lines at the same three moments in time. The second calculation used the spatial adaptation procedures starting with the coarse mesh of the first calculation. The mesh was locally pre-embedded about the initial pressure discontinuity for two levels of enrichment in order for the initial discontinuity to be sharply defined. The solution was marched in time for 3,000 time steps and the mesh was adapted to the magnitude of the density gradi-
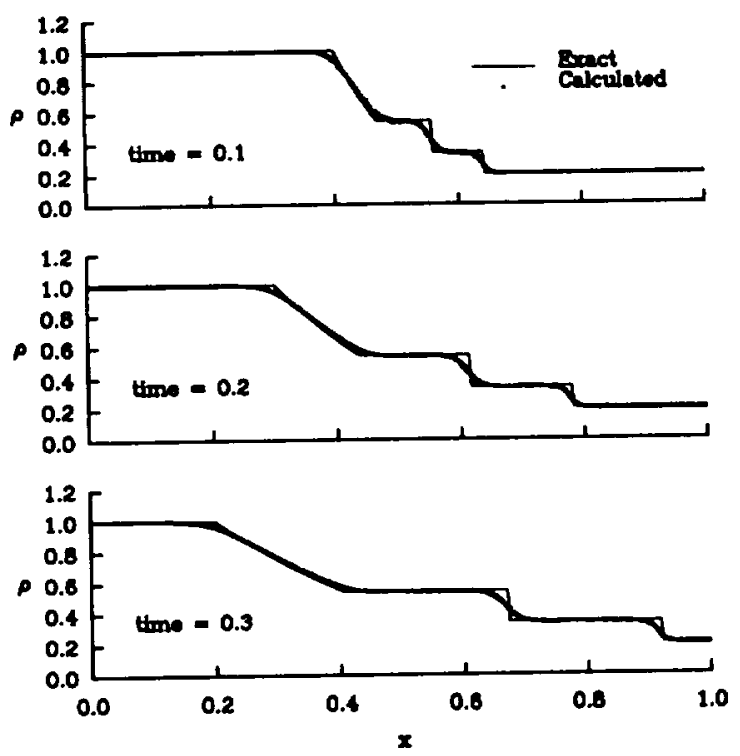

Fig. 20 Comparison of calculated and exact density profiles in a shock-tube for a sequence of times $(0.1,0.2,0.3)$ on the original coarse mesh (every other point plotted).

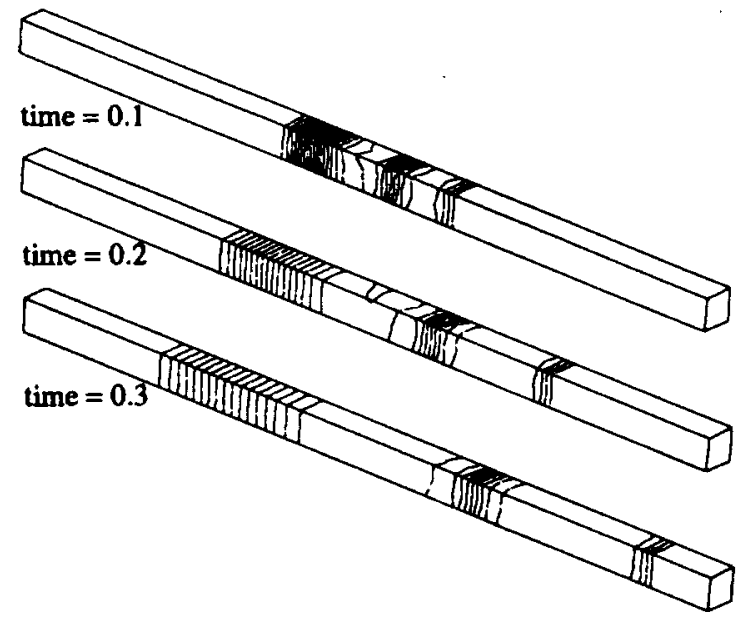

Fig. 21 Surface density contour lines $(\Delta \rho=0.0067)$ for a shock-tube for a sequence of times $(0.1,0.2$, $0.3)$ using the original coarse mesh.

ent once every 20 time steps using a threshold value of 1.0. During the course of the calculation, mesh refinement was restricted to two levels of enrichment. Figure 22 shows the resulting density profiles using spatial adaptation for the same three moments in time that were shown previously. Similar to the previous results, these profiles were obtained by sorting all the cell-centered values of the primitive variables according to their $\mathbf{x}$-coordinate locations and plotting every 20th point. In this figure the amount of adaptation can be seen in the distribution of the calculated data throughout the tube, where the majority of the cells are concentrated in the regions of the expansion fan, the contact surface, and the shock wave. The concentration of cells can be seen more
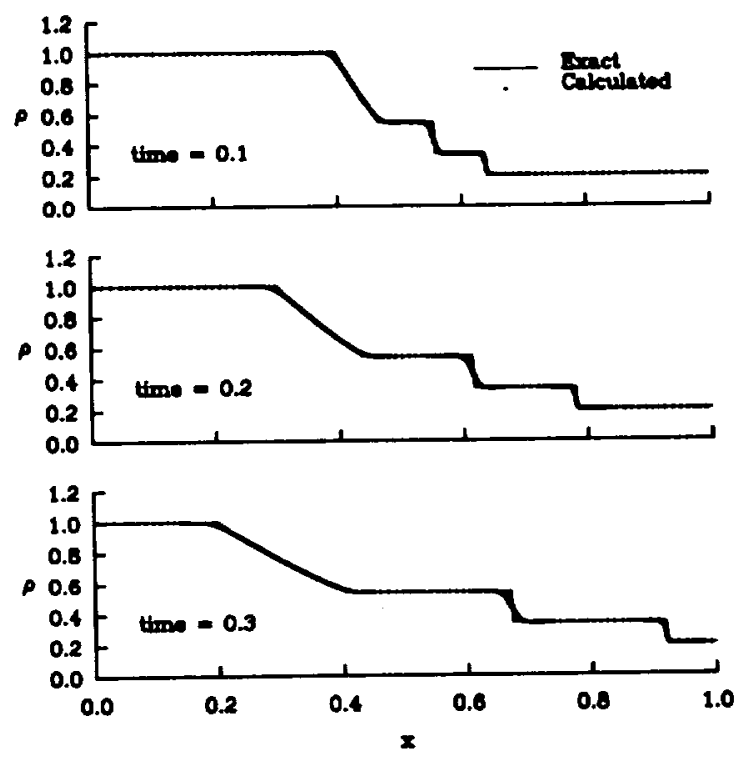

Fig. 22 Comparison of calculated and exact density profiles in a shock-tube for a sequence of times $(0.1,0.2,0.3)$ on the adapted mesh (every 20 th point plotted). 

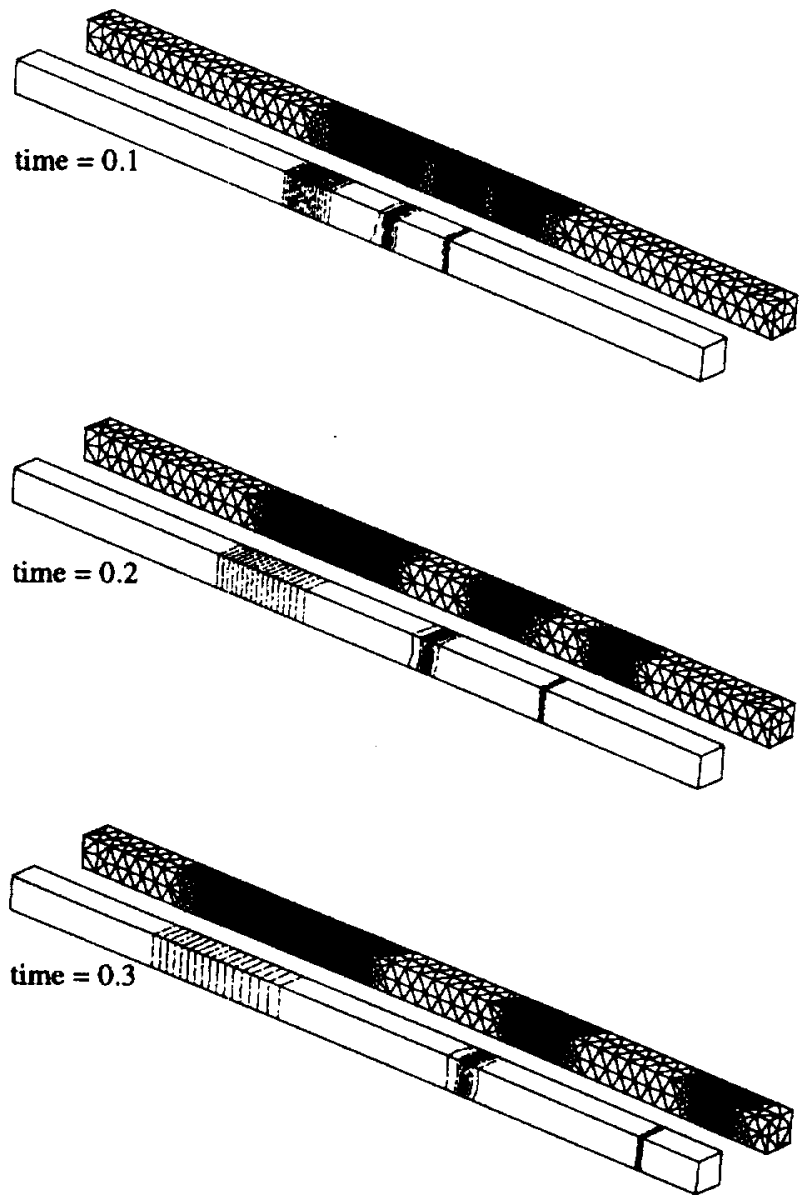

Fig. 23 Surface mesh and density contour lines $(\Delta \rho=0.0067)$ for a shock-tube for a sequence of times $(0.1,0.2,0.3)$ using an adapted mesh.

clearly in Fig. 23 where the surface mesh and surface density contour lines are shown. The figure illustrates, by comparison with Fig. 21, that the shock wave is sharply captured using the spatial adaptation procedures. Figure 22 shows good agreement in temporal and spatial accuracy with the exact solution giving confidence in the adaptive mesh procedures. The shock-tube results obtained using the spatial adaptation procedures required approximately 5.5 hours of CPU time and $18.2 \mathrm{mw}$ of memory on the Cray-2s computer at NASA LaRC. Also, the spatial adaptation procedures consumed approximately $20 \%$ of the total CPU time.

\section{Concluding Remarks}

Spatial adaptation procedures for the accurate and efficient solution of steady and unsteady inviscid flow problems were described. The adaptation procedures were developed and implemented within a three-dimensional unstructuredgrid upwind-type Euler code. These procedures involve mesh enrichment and mesh coarsening to either add points in high gradient regions of the flow or remove points where they are not needed, respectively, to produce solutions of high spatial accuracy at minimal computational cost.

Steady and unsteady results were presented to demonstrate applications of the spatial adaptation procedures to three-dimensional problems. Steady transonic flow results were obtained for the ONERA M6 wing to assess the accuracy of the computed surface pressures by making comparisons with experimental data. The results obtained using spatial adaptation were found to be in good agreement with the experimental data giving confidence in the mesh enrichment and coarsening procedures. Unsteady flow results were obtained in a three-dimensional simulation of a one-dimensional shock-tube problem to demonstrate an application of the mesh enrichment and coarsening procedures for a time-dependent problem. The accuracy of the computed results was assessed by making comparisons with the exact solution. Both the steady and unsteady solutions obtained using spatial adaptation were shown to be of high spatial accuracy, primarily in that the shock waves were sharply captured.

\section{Acknowledgments}

The work constitutes a part of the first author's Ph.D. thesis at Purdue University and was supported by the NASA Graduate Student Researchers program under grant NGT50406. The authors would like to thank Paresh Parikh and Shahyar Pirzadeh of ViGYAN, Inc., Hampton, Virginia for providing the mesh generation program that was used to generate the three-dimensional meshes in the present study. The authors would also like to thank Robert Neely of Lockheed Engineering and Sciences Company for providing a beta version of the Flow Analysis Software Toolkit (FAST) to view the unstructured meshes and solutions.

\section{References}

[1] Thompson, J. F., "A Survey of Dynamically-Adaptive Grids in the Numerical Solution of Partial Differential Equations," AIAA Paper No. 84-1606, June 1984.

[2] Löhner, R., "Finite Elements in CFD: What Lies Ahead," International Journal for Numerical Methods in Engineering, vol. 24, pp. 1741-1756, 1987.

[3] Nakahashi, K., and Deiwert, G. S., "Self-AdaptiveGrid Method with Application to Airfoil Flow," AIAA Journal, vol. 25, pp. 513-520, Apr. 1987.

[4] Usab, W. J., and Murman, E. M., "Embedded Mesh Solution of the Euler Equation Using a Multiple-Grid Method," AIAA Paper No. 83-1946-CP, July 1983.

[5] Dannenhoffer, J. F., and Baron, J. R., "Grid Adaption for the 2-D Euler Equations," ALAA Paper No. 850484, Jan. 1985. 
[6] Dannenhoffer, J. F., and Baron, J. R., "Robust Grid Adaption for Complex Transonic Flows," AIAA Paper No. 86-0495, Jan. 1986.

[7] Peraire, J., Vahdati, M., Morgan, K., and Zienkiewicz, O. C., "Adaptive Remeshing for Compressible Flow Computations," Journal of Computational Physics, vol. 72 , pp. $449-466$.

[8] Peraire, J., Morgan, K., Peiro, J., and Zienkiewicz, O. C., "Adaptive Finite Element Method for High Speed Flows," AIAA Paper No. 87-0558, Jan. 1987.

[9] Löhner, R., "Adaptive H-Refinement on 3-D Unstructured Grids for Transient Problems," AIAA Paper No. 89-0365, Jan. 1989.

[10] Kallinderis, Y., Parthasarthy, V., Wu, J., "A New Euler Scheme and Adaptive Refinement/Coarsening Algorithm for Tetrahedral Grids," AIAA Paper No. 920446, Jan. 1992.

[11] Rausch, R. D., Batina, J. T., and Yang, H. T. Y., "Euler Flutter Analysis of Airfoils Using Unstructured Dynamic Meshes," Jourmal of Aircraft, vol. 27, no. 5, pp. 436-443, 1990.

[12] Rausch, R. D., Batina, J. T., and Yang, H. T. Y., "Spatial Adaption Procedures on Unstructured Meshes for Accurate Unsteady Aerodynamic Flow Computation," AIAA Paper No. 91-1106, Apr. 1991.

[13] Batina, J. T., "Three-Dimensional Flux-Split Euler Schemes Involving Unstructured Dynamic Meshes," AIAA Paper No. 90-1649, June 1990.

[14] Rausch, R. D., Batina, J. T., and Yang, H. T. Y., "ThreeDimensional Time-Marching Aeroelastic Analyses Using an Unstructured-Grid Euler Method," AIAA Paper No. 92-2506, Apr. 1992.

[15] Rausch, R. D., Time-Marching Aeroelastic and Spatial Adaptation Procedures on Triangular and Tetrahedral Meshes Using an Unstructured-Grid Euler Method. PhD thesis, Purdue University, Dec. 1992.
[16] Van Leer, B., "Flux-Vector Splitting for the Euler Equations," Lecture Notes in Physics, vol. 170, pp. 507512, 1982.

[17] Parikh, P., Pirzadeh, S., and Löhner, R., "A Package for 3-D Unstructured Grid Generation, Finite-Element Flow Solution and Flow Field Visualization," NASA CR-182090, Sept. 1990.

[18] Anderson, W. K., Thomas, J. L., and Whitfield, D. L., "Three-Dimensional Multigrid Algorithms for the FluxSplit Euler Equations," NASA TP-2829, NASA Langley Research Center, Nov. 1988.

[19] Batina, J. T., "Accuracy of an Unstructured-Grid Upwind-Euler Algorithm for the ONERA M6 Wing," Joumal of Aircraft, vol. 28, June 1991.

[20] Woodard, P. R., Batina, J. T., and Yang, H. T. Y., "Quality Assessment of Two- and Three-Dimensional Unstructured Meshes and Validation of an Upwind Euler Flow Solver," ALAA Paper No. 92-0444, Jan. 1992.

[21] Halt, D. W., A Compact High-Order Euler Solver for Unstructured Grids. PhD thesis, Sever Institute of Washington University, May 1992.

[22] Schmidt, V. and Charpin, F., "Pressure Distributions on the ONERA M6 Wing at Transonic Mach Numbers," Appendix B1 in AGARD-AR-138, Experimental Data Base for Computer Program Assessment, May 1979.

[23] Frink, N. T., Parikh, P., and Pirzadeh, S., "A Fast Upwind Solver for the Euler Equations on ThreeDimensional Unstructured Meshes," ALAA Paper No. 91-0102, Jan. 1991.

[24] Mavriplis, D. J., "Three Dimensional Unstructured Multigrid for the Euler Equations," ALAA Paper No. 91-1549, June 1991.

[25] Liepmann, H. W., and Roshko, A., Elements of Gasdynamics. New York: John Wiley and Sons Inc., 1957. 


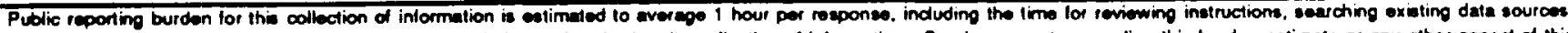

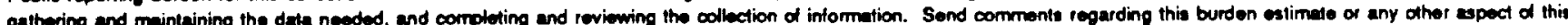

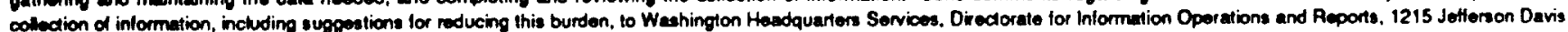
Highway, Sulle 1204. Alington. VA 22202-4302, and to the Otlice of Management and Budget, Peporwork Reduction Project (0704-0188), Washington, DC 20503.

\begin{tabular}{|l|l|l}
\hline 1. AGENCY USE ONLY (Laevo blank) & $\begin{array}{l}\text { 2. REPOAT DATE } \\
\text { February } 1993\end{array}$ & $\begin{array}{l}\text { 3. REPORT TYPE AND DATES COVERED } \\
\text { Technical Memorandum }\end{array}$ \\
\hline
\end{tabular}

\section{TITLE AND SUBTTLE}

Spatial Adaptation Procedures on Tetrahedral Meshes for Unsteady Aerodynamic Flow Calculations

$$
\text { 505-63-50-12 }
$$

6. AUTHOR(S)

Russ D. Rausch

John T. Batina

Henry T. Y. Yang

7. PERFOAMINO ORGANIZATION NAME(9) AND ADDRESS(ES)

NASA Langley Research Center

8. PERFORMING ORGANIZATION

Hampton, VA 23681-0001

9. SPONSORING / MONITORING AGENCY NAME(S) AND ADDRESS(ES)

National Aeronautics and Space Administration

Washington, DC 20546-0001
10. SPONSOAING / MONITOAING ACENCY AEPOAT NUMBEA

NASA TM 107726

\section{SUPPLEMENTARY NOTES}

Paper Presented at the AIAA 31st Aerospace Sciences Meeting, Reno, Nevada, January 11-14, 1993.

12n. DISTAIBUTION I AVAILABILTYY STATEMENT

Unclassitied - Unlimited

Subject Category 02 12b. DISTRIBUTION CODE

\section{ABSTAACT (Maximum 200 worda)}

Spatial adaptation procedu : es for the accurate and efficient solution of steady and unsteady inviscid flow problems are described. The adaptation procedures were developed and implemented within a three-dimensional, unstructured-grid, upwind-type Euler code. These procedures involve mesh enrichment and mesh coarsening to either add points in high gradient regions of the flow or remove points where they are not needed, respectively, to produce solutions of high spatial accuracy at minimal computational cost. The paper gives a delailed description of the enrichment and coarsening procedures and presents comparisons with experimental data for an ONERA M6 wing and an exact solution for a shock-tube problem to provide an assessment of the accuracy and efficiency of the capability. Steady and unsteady results, obtained using spatial adaptation procedures, are shown to be of high spatial accuracy, primarily in that discontinuities such as shock waves are captured very sharply.

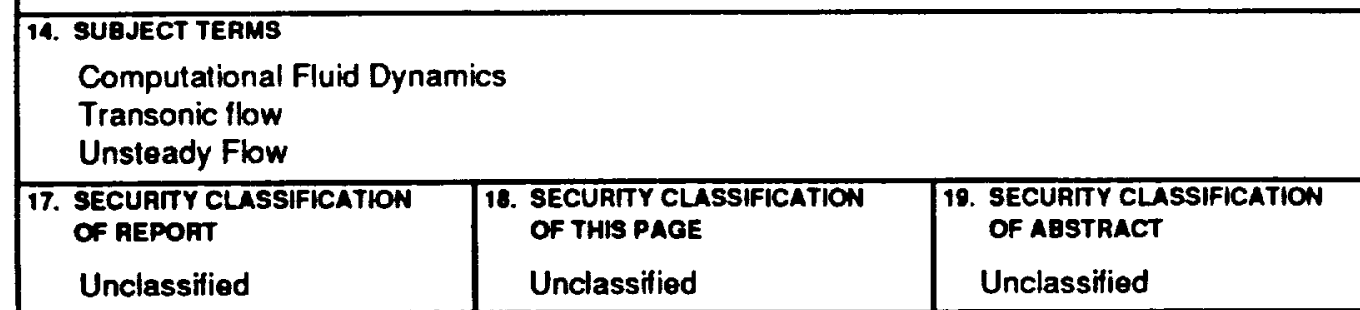

15. NUMBEA OF PAGES

14

16. PAICE CODE $\mathrm{AO} 3$

20. UMTTATION OF ABSTRACT

